\title{
Direction-specific motion thresholds for abnormal image shifts during saccadic eye movement
}

\author{
WILLIAM R. WHIPPLE \\ University of California, San Diego, LaJolla, California 92037 \\ and \\ HANS WALLACH \\ Swarthmore College, Swarthmore, Pennsylvania 19081
}

\begin{abstract}
Eye movements were monitored and a target circle subtending an angle of $7^{\circ}$ was made to move during and dependent on the eye movements. Thresholds of detection of the resulting abnormal image displacements were obtained. Thresholds were low when both the eyes and the target moved either horizontally or vertically. They were higher by a factor of two or more when the eye movements and the target motions were not in the same plane. In the latter conditions, two processes account for the detection of target motion. One is a compensation process where the extent of that component of the motion of the retinal image of the target which is parallel to the eye movement is compared with the extent of the eye movement. The other process detects an angle between the plane of the target image motion and the plane of the eye movement. Our results indicate that the higher thresholds occurred when detection of this angle was required.
\end{abstract}

Whenever we move our eyes, the images projected on our retinae shift position. But, despite these image shifts, the visual field appears to remain stationary. By contrast, should there be an image shift while the eyes remain motionless, we see something moving. Some process must enable us to differentiate between these two causes of retinal image displacement. Little is known about the nature of this process. It has often been assumed to be an accounting mechanism, which matches up the size of an eye movement with the simultaneously occurring image displacements (Kaufman, 1974, pp. 372-374; Kling \& Riggs, 1971, pp. 530-532). An accounting mechanism of this type is known to be responsible for the stability of the perceived environment during head movements (Wallach \& Kravitz, 1965, 1968).

An experiment by Mack (1970) yielded data which argue in favor of such a mechanism. Mack used electrooculography to monitor flash-induced horizontal saccades in subjects who were watching a luminous point in an otherwise dark field. The output of the eye-movement monitor caused the target to move, either vertically or horizontally, as long as the eye was in motion. Mack found that target shifts amounting to more than about $20 \%$ of the size of

This work was supported by Grant 11089 from the National Institute of Mental Health to Swarthmore College, Hans Wallach, principal investigator. We are most grateful to V. R. Carlson for his help in preparing this report. the eye's movement were reliably interpreted as target motion, and that this threshold depended scarcely at all upon the direction of the target's objective displacement-with the eye (target moving left as the eye moves left), against the eye (target moving right as the eye moves left), or even orthogonal (target moves up or down as the eye moves to the left).

Mack's experiment suggests that subjects can, with reasonable accuracy, detect retinal image displacements that are larger or smaller than the concomitant eye movements would warrant. In contrast to this, Wallach and Lewis (1966) reported results which suggested extremely poor accuracy at such a task. They used an optical technique to produce abnormal image displacements during eye movements; the target appeared to the subject as a large disk, which was inspected by free eye movements. The subjects frequently failed to detect abnormal image displacement, even when it was more than $100 \%$ larger than what would have been normal. Wallach and Lewis interpreted this finding in terms of a discounting model, according to which visual processing ignores retinal image shifts which take place during eye movements.

The striking difference between these two results was one of the reasons for our present experiment. We wondered, in particular, whether Mack's finding of fairly accurate detection of abnormal image shifts during eye movements was peculiar to her experimental conditions or whether it could be ob- 
tained in a situation more similar to that of Wallach and Lewis, with a larger target and free (rather than flash-induced) eye movements. Mack's report that the threshold of motion was essentially the same when the target motion was orthogonal to the eye movement rather than congruent with it seemed to us surprising.' We, therefore, wanted to explore further conditions in which the target's displacement and the eye's movement were not congruent, that is, where the target moved in a plane other than that of the eye's rotation. Such conditions result in a displacement of the target's image on the retina whose direction is different from that of an image displacement due solely to an eye movement.

\section{METHOD}

\section{Subjects}

The subjects were eight paid undergraduates (five male, three female), selected for the ease with which the critical alignment of the apparatus could be obtained. There were several details of facial structure which made alignment more or less difficult: the size and spacing of the eyes, the color of the iris, the shape and size of the nose, and the normal position of the lids. In all, we were able to complete alignment with about half of those who responded to our advertisements. All of the subjects in the main experiment and subsequent controls had participated in one or more pilot experiments using the same apparatus and similar techniques. ${ }^{2}$

\section{Apparatus and Procedure}

We measured subject's eye movements with an infrared reflection technique, using a Biometrics SGH/V-2 eye-movement monitor. ${ }^{3}$ This device simultaneously monitors both horizontal and vertical eye movements by amplifying the signal from carefully aimed, differentially connected photoreceptors at the sclerocorneal boundary (for horizontal movements) or at the eyelid edge (for vertical movements). In our setup, horizontal movements were measured from the right eye and vertical movements from the left. The very critical positioning of the photoreceptors was maintained by fixing the subject's head with a biteboard and a headrest; the photoreceptors were mounted on micromanipulators to make accurate adjustment possible.

The apparatus was calibrated individually for each subject. During calibration, the output of the monitor controlled the position of the blip on an ocscilloscope screen while the subject fixated, at the experimenter's instruction, the numbered marks of a luminous calibration display. A scale replica of this display was marked on the oscilloscope screen, which the experimenter could see but which the subject could not. The experimenter adjusted the apparatus until the blip's position always corresponded with the mark being fixated by the subject. This empirical calibration was very time-consuming, but it enabled us to obtain highly accurate following of the subject's eye movements.

After alignment was complete, the calibration display in front of the subject was replaced by a second cathode-ray tube (CRT) which was an extension of the CRT in the experimenter's oscilloscope. The subject's CRT screen was not marked and had a special fast-decay phosphor to minimize any aftertrace. A blink button, controlled by the subject, enabled him to blank out his screen whenever he had difficulty maintaining fixation; the experimenter also could blank out the subject's screen. In all other respects, the subject's CRT screen showed exactly the same display that was shown on the experimenter's screen.

After calibration, both screens showed a dim ring, produced with the oscilloscope by a Lissajous technique. The ring, which for the subject subtended an approximately $7^{\circ}$ visual angle, was displaced in accordance with the subjects eye movements, just as the blip had been during calibration. The experimenter could vary the amount of the ring's displacement relative to the eye's movement by adjusting a calibrated attenuator connected to the output of the eye-movement monitor. This ratio (target motion/eye movement) will be called displacement ratio (DR) and expressed as a percent. A target that remains stationary regardless of subject's eye movement has a DR of 0\%. A target that moves the same amount as the eye has a DR of $100 \%$; if it moved in the same direction as the eye, such a target would produce an image stabilized on the retina. In this experiment, we used DRs ranging from $0 \%$ to $40 \%$.

We took considerable care that, during the experiment proper, the ring-shaped target was the only object visible to the subject. This was difficult, as the experimental sessions were long (so that subjects became totally dark-adapted) and the stimulus was produced on a cathode-ray tube, which inevitably emits some stray light. The special CRT, custom manufactured for this experiment, has a very fast decay phosphor and a very low level of stray light emission. We kept screen illumination as low as possible, but since our task required foveal fixation, purely scotopic levels were not feasible. We placed filters in front of the screen which passed light at the wavelength of the target, while greatly reducing stray light from the screen. Finally, we used our subjects as an additional check. During pilot sessions (in which all subjects participated), they were encouraged to report, at any point in the experiment, any light they could detect from a source other than the stimulus. In several cases, they did make such reports; we tracked down the source in each case (usually reflection from a part of the apparatus of light which escaped from around the edges of the filters) and provided baffles or masks to eliminate the problem. During the main experiment, it is certain that only the target was visible to the subjects.

During calibration, the blip had always moved in a direction congruent with the eye's movement. But after alignment, when the blip had been replaced with the ring, orthogonal or oblique target displacements could easily be set up. For example, when the output from the eye-movement monitor's horizontal channel was connected to the oscilloscope's vertical channel, horizontal eye movements caused vertical target displacement; this orthogonal condition corresponds to the one used by Mack. Similarly, vertical eye movements could be made to cause horizontal target displacements. Again, if the output of the monitor's horizontal channel was connected to both the vertical and the horizontal channels of the oscilloscope, a horizontal eye movement produced a target displacement that was oblique, at a $45^{\circ}$ angle from the vertical or horizontal plane. By manipulations of this sort, we could arrange for target movements in horizontal, vertical, or oblique directions to be caused by eye movements in any of the same three directions.

When eye and target movements were congruent, the target always moved with the subject's eye movements; that is, it moved to the left if the eyes moved to the left. Mack's data, as well as pilot data of our own, suggested that the opposite condition (where the target moves right for eye movements to the left) yields results very similar to those for the with condition; to simplify the experimental design, we omitted this as a variable. In orthogonal eye/target conditions, the directions were arbitrarily assigned and differed for different observers (for example, an upward eye movement caused the target to move toward the left for some observers, toward the right for others). When an oblique target movement was caused by a nonoblique eye movement, the component of the oblique movement that was congruent always moved with the eye; an upward eye movement sometimes caused a target movement up and to the right, sometimes up and to the left, but never in any downward direction.

After alignment was completed, the subjects began the series of experimental trials. Before each trial, the experimenter in- 
structed the subject to look back and forth from one edge of the ring to its opposite, in one of three directions: horizontal, vertical, or oblique; in the latter case, the eye movements were either from upper left to lower right or from upper right to lower left. The two types of oblique movements were counterbalanced throughout the experiment.

The subject was told to fixate steadily one edge of the ring before shifting his gaze to the opposite side, "as though you were comparing the two edges of the ring to see whether they were equally sharp." $4 \mathrm{He}$ was allowed to determine the rate and number of eye movements that would seem natural for him in such a task; generally, six to eight saccades were made on each observation, at a rate of 1 to $2 / \mathrm{sec}$.

After the subject had made his observation, he terminated the trial by blanking the CRT screen, and reported the direction of the ring's displacement, if any was seen. The subject made reports by pressing buttons on a panel attached to the arm of his chair; these illuminated lamps on a display panel which only the experimenter could see. This technique meant that the subject could report on a trial without leaving the biteboard; but it placed two constraints on his reports. He had to select one of the four planes in which the target could have actually movedhorizontal, vertical, or the two oblique planes. Also, the subject could not report the direction of the target's apparent movement (whether it moved with or against the eyes) but only the plane in which it seemed to move. The subject chose the one of the four possible planes which came closest to the target's perceived path. A special button allowed the subject to report no perceived object motion.

As previously described, our apparatus made it possible for any one of three types of eye movement to produce any one of three possible types of target displacement. Thus there were nine eye-movement/target-motion combinations. (The two oblique directions were considered as a single combination.) Each subject was presented with all nine combinations, each of which was given with nine different displacement ratios, from $0 \%$ to $40 \%$ in steps of $5 \%$ DR.

In cases involving oblique eye or target movements, the $5 \%$ DR steps are only nominal, being defined with respect to the horizontal or vertical component of the motion path. The effective displacement ratio is found by vector arithmetic, and these effective values were used in Figure 1 and the data analyses. There were 81 conditions in all, and each subject went through the entire series twice, in different random orders. The procedure took from 4 to $7 \mathrm{~h}$. The subject was permitted to rest his eyes as needed between trials, and extended breaks were taken from time to time.

\section{RESULTS}

The nine cells in Figure 1 are concerned with each of the nine combinations of eye movement direction and target motion direction. In each cell, the percent correct responses obtained from all subjects are plotted against the percent DR of the target motions. A correct response was one on which the subject correctly reported whether the target moved or not and was also correct concerning the plane in which the target moved. Both the filled and the open circles represent data points, each the mean of 16 trials. The open circles indicate uninformative data points, either displacement ratio values at which the subjects reported correctly only at approximately chance levels $(\mathbf{2 0 \%})$ or displacement ratio values at which they were $100 \%$ correct. These uninformative data

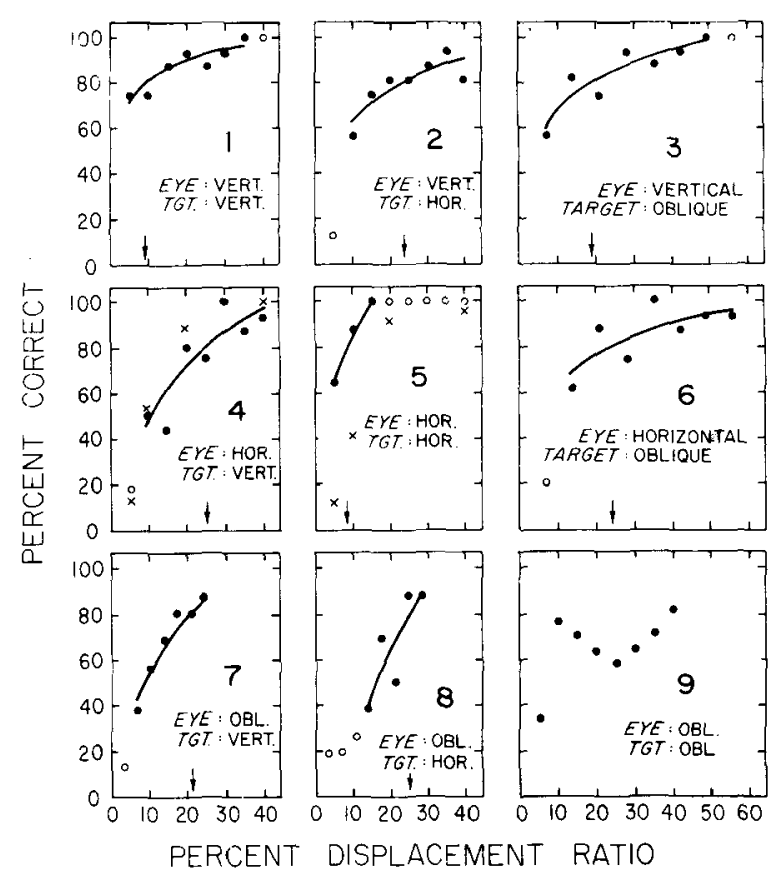

Figure 1. Percent correct identification of target motion direction as a function of displacement ratio (DR) for nine eyemovement-direction/target-movement-direction conditions. Open circles represent data excluded from threshold calculations (see text); crosses represent data from Mack's (1970) experiment; arrows indicate the $80 \%$ thresholds.

points were omitted from the calculations to be presented below.

The curves shown in the nine cells of Figure 1 are the best-fitting logarithmic curves for the data points. In most cases, logarithmic curves gave a much better fit than any other simple monotonic curve. In Cells 1 and 8 , linear equations also gave a good fit (as did a power equation in Cell 8), but it was not possible to decide between these different kinds of equations on the basis of mean squared residuals, because they were nearly identical. We chose logarithmic curves for these cells because curves of this form were clearly superior in the case of the other cells. Since the uninformative data points (open circles) were not used in calculating the regression equations, the corresponding curves are shown only in the domain of informative data points. No curve is shown for Cell 9, since the data points suggest that no simple monotonic function would be appropriate.

Table 1 gives the equations that correspond to the curves in Cells 1-8 of Figure 1. By substitution, thresholds of motion can be calculated from these equations. We computed the $80 \%$ thresholds-that is, the percent DR for each condition at which subjects may be expected to report correctly the direction of motion $80 \%$ of the time. These $80 \%$ thresholds are also given in Table 1 , and are in- 
Table 1

Best-Fitting Logarithmic Equations for Data Points in Figure 1 and $80 \%$ Thresholds of Motion

\begin{tabular}{cccccc}
\hline Cell & Eye & Target & Equation & $\begin{array}{c}80 \% \\
\text { Threshold } \\
\text { (\%DR) }\end{array}$ \\
\hline 1 & V & V & $\mathrm{y}=29.074 \log \mathrm{x}+51.798$ & 9.3 \\
2 & $\mathrm{~V}$ & $\mathrm{H}$ & $\mathrm{y}=47.865 \log \mathrm{x}+14.512$ & 23.3 \\
3 & $\mathrm{~V}$ & $\mathrm{O}$ & $\mathrm{y}=46.254 \log \mathrm{x}+20.170$ & 19.7 \\
4 & $\mathrm{H}$ & $\mathrm{V}$ & $\mathrm{y}=88.005 \log \mathrm{x}-43.530$ & 25.3 \\
5 & $\mathrm{H}$ & $\mathrm{H}$ & $\mathrm{y}=78.813 \log \mathrm{x}+7.830$ & 8.2 \\
6 & $\mathrm{H}$ & $\mathrm{O}$ & $\mathrm{y}=46.699 \log \mathrm{x}+15.312$ & 24.3 \\
7 & $\mathrm{O}$ & $\mathrm{V}$ & $\mathrm{y}=80.019 \log \mathrm{x}-25.990$ & 21.1 \\
8 & $\mathrm{O}$ & $\mathrm{H}$ & $\mathrm{y}=157.950 \log \mathrm{x}-141.264$ & 25.2 \\
9 & O & O & & & \\
\hline
\end{tabular}

Note-Letters $H, V$, and $O$ represent horizontal, vertical, and oblique movement, respectively. No monotonic function gives a satisfactory fit to the condition in Cell 9.

dicated in Figure 1 as well by small arrows above the abscissae. These thresholds show that subjects were very accurate at recognizing congruent target motions when the eye movements were cardinal, that is, horizontal or vertical. These thresholds were at $8.2 \%$ and $9.3 \%$ DR, respectively. All six conditions where the direction of eye movement and the plane of the target motion differed, the noncongruent conditions of Cells $2,3,4,6,7$, and 8 had much higher thresholds which agreed with each other rather well. These thresholds ranged from $19.7 \%$ to $25.3 \%$ DR. Strikingly different from these results was the threshold obtained for the case of oblique congruent eye-target displacement (Cell 9). Here a threshold could not be calculated, but the fact that there were only two errors at the level of $40 \%$ target motion makes it likely that the threshold lies between $40 \%$ and $50 \%$. This is in complete disagreement with the other two congruent target motion conditions (Cells 1 and 5), where the thresholds were the lowest.

The high threshold in the oblique congruent condition caused us to wonder whether the oblique eye movements made by our subjects were different in form from cardinal movements. In making an oblique eye movement, the subject's eyes may have made an alternating series of small horizontal and vertical movements, rather than a smooth oblique movement. Such a discontinuity in the oblique eye movements could have affected the threshold. It also seemed possible that the horizontal and vertical components of an oblique eye movement, even if not discontinuous, might differ from each other in latency; the threshold could be affected by such differences.

We checked these possibilities in a control experiment in which the outputs of the two channels of the eye-movement monitor were connected to the two channels of a Beckman polygraph, so that a permanent record of the eye's behavior could be ob- tained. Three of the subjects from the main experiment served again; they made eye movements in horizontal, vertical, and both of the oblique directions, with both stationary and displacing targets. (The displacing targets moved in a congruent direction at $30 \%$ DR.) The polygraph records showed no discontinuity in the oblique eye movements, and no indication of a latency difference. Except for the fact that both channels were active, the oblique eye-movement records were indistinguishable from those from cardinal eye movements.

It is possible to make direct comparisons between the thresholds obtained by Mack and those obtained under corresponding conditions. Mack used conditions corresponding to those of Cells 4 and 5 of Figure 1. In these cells, her data have been plotted as Xs. When we treated her data in the same manner we treated our own, by fitting logarithmic curves and finding the $80 \%$ thresholds, we obtained thresholds of $22.3 \% \mathrm{Dr}$ for her condition corresponding to Cell 4 , and $23.9 \%$ DR in the condition similar to that in our Cell 5. The threshold calculated from Mack's data in Cell 4 closely resembles the $25.3 \%$ DR which we obtained; but the 23.9\% DR threshold calculated from Mack's data is substantially higher than our corresponding threshold of $8.2 \% \mathrm{DR}$.

\section{DISCUSSION}

Our results support previous findings by Mack that an accounting mechanism operates in the perception of stationary objects during eye movements. We found that congruent target motions during horizontal eye movements were perceived with good accuracy. Our congruent target motion decreased the normal displacement that the retinal image of a stationary object undergoes during an eye movement. When that decrease exceeded threshold value, the target motion was perceived. The nervous system evaluates the amount of image displacement during eye movements with fair accuracy, for we obtained a threshold value of $8.2 \%$ DR (Condition 5), which was even lower than the one reported by Mack. Congruent target motion during vertical eye movements also had a low threshold, just under $10 \%$ DR (Condition 1).

Our results provide some information about the reason for the extremely poor detection of abnormal image shifts during eye movement that Wallach and Lewis reported. ${ }^{5}$ In contrast to Mack, we used free eye movements and an extended target, as did Wallach and Lewis. Nevertheless, we obtained low thresholds for the detection of congruent target motion. The only remaining difference between our experiment and that of Wallach and Lewis was the very much greater size of their target. We believe 
that the difference in the visual angle of the target is responsible for the different results. Unfortunately, it was not possible to support this interpretation by experimenting with intermediate sizes of target image. Our own techniques would not yield a circle of a substantially larger visual angle, and the target used by Wallach and Lewis, the projection of the pupil on the retina by a very divergent light beam, could not be made smaller without loss of its abnormal displacements during eye movements.

The thresholds for noncongruent target motions from Cells 2, 3, 4, 6, 7, and 8 ranged from $19.7 \%$ to $25.3 \%$ DR and were more than twice as high as the threshold for the two congruent conditions just discussed. The fact that these thresholds differ from those for congruent target displacements is, however, not surprising, for the detection of such noncongruent target displacements has a different basis from that of congruent target displacement. Unlike the congruent case, target motion in a plane that differs from the plane of the eye's movement affects the direction of the image displacement on the retina. Figure $2 \mathrm{~A}$ illustrates what happens in Condition 4 (horizontal eye movement and vertical target motion). As the eye moves from $O$ to $E$, the retinal image of a stationary target would be displaced by the same amount in the opposite direction; that is, from O to I. However, in Condition 4, the eye movement has been made to cause the target to move in a plane perpendicular to the path of the eye's movement, upward in this example. The length of target motion vector OT would differ for different displacement ratios. (The illustraton is drawn for the case of $30 \%$ DR.) The resultant retinal path of the target image is represented by vector $O R$, and the angle between OR and the plane of the eye's movement is shown as $\theta$. The size of angle $\theta$ may be calculated by using the equation, $\tan \theta=I R / O I$.

If $O I$ is defined as having the value 1.0 , then IR, because it is equal to OT, will be equal to the displacement ratio. Thus, in Condition 4 , the limiting values of $\theta$ are $2.9^{\circ}$ for a vertical target displacement of $5 \% \mathrm{DR}$ and $21.8^{\circ}$ for vertical target displacement of $40 \%$ DR. Condition 2 (vertical eye movement, horizontal target motion) corresponds to Condition 4 , and the calculation of the angle is the same. At the threshold values of $25.3 \%$ DR for Condition 4 and of $23.3 \%$ DR for Condition 2, the angle $\theta$ amounted to $14.2^{\circ}$ and $13.1^{\circ}$, respectively.

Correct target motion reports in a case of orthogonal target displacements must result from a target image path that deviates by the angle $\theta$ from the horizontal or vertical image path that is normally associated with horizontal or vertical eye movements. The compensating mechanism that prevents perception of congruent target motion when the image of a stationary target is displaced during an eye move- ment must, however, also be in operation. A target that moves vertically during a horizontal eye movement also undergoes a horizontal displacement relative to the horizontally moving eye; that displacement is the same as that of a stationary target. If this horizontal displacement in the direction opposite to the eye movement is not perceived, the compensating mechanism must be in operation. Since the objective vertical displacement of a target during a horizontal eye movement is given as an oblique target image path, the compensating mechanism eliminates the effect of the horizontal component of the oblique target image motion leaving the vertical component to be perceived. Without the elimination of the horizontal component of the oblique target image motion so that only the vertical component remains, the reports on perceived target motion should have been preponderantly horizontal. (The angle $\theta$ that the retinal image path forms with horizontal varied between $2.9^{\circ}$ and $21.8^{\circ}$ for the range of displacement ratios of $5 \%$ to $40 \%$ DR offered, and the path was therefore always closest to the horizontal direction.) Corresponding considerations apply to Condition 2, where the target motion was also orthogonal to the plane of the eye movements.

Figure 2A makes it clear that the angle $\theta$ depends on OT when OI remains unchanged; if OT is made larger, $\theta$ becomes larger. That means that the angle $\theta$ varies with the extent of the vertical target motion relative to the eye movement, that is, with the value of its displacement ratio. We must keep this in mind when we consider how objectively oblique target displacement is distinguished from vertical target motion. The thresholds for oblique target motion of $19.7 \%$ and $24.3 \%$ DR measured
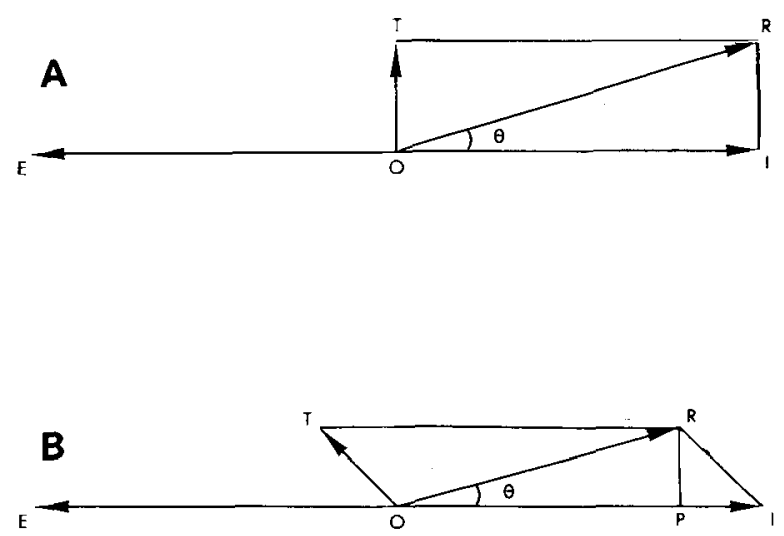

Figure 2. Retinal path of target image in representative conditions. Drawing A illustrates Condition 4: OE represents the eye movement, OI the displacement of a stationary target on the retina, OT the artificial displacement produced by the apparatus, yielding a resultant retinal path $O R$. The significance of angle $\theta$ is discussed in the text. Drawing $B$ shows the same vectors for Condition 6. 
in Conditions 3 and 6 show that oblique target motion is easily distinguished from vertical target motion. Both are, of course, represented on the retina by oblique target image paths. For oblique target motion, the angle $\theta$ of that path is only slightly smaller than for vertical target motion of the same DR value. But even if that difference were much larger, it would not be of help. Since in both cases the angle $\theta$ depends on the value of the displacement ratio, the distinction between objectively vertical or oblique target motion cannot be based on the size of the angle $\theta$.

The following consideration shows how orthogonal target motion (Conditions 2 and 4) can be distinguished from oblique target motions (Conditions 3 and 6). Figure 2B illustrates Condition 6, where the target moves at an angle $45^{\circ}$ to the eye's horizontal movement. The target motion vector OT therefore forms an angle of $45^{\circ}$ with the path OI of the target image due to eye movement. The resultant retinal path $O R$ is formed as the diagonal in the parallelogram OIRT. Angle $\theta$ may be calculated by drawing a vertical line through point $R$, intersecting $\mathrm{OI}$ at point $\mathrm{P}$. Since angle PIR is $45^{\circ}$, we have

$$
\begin{gathered}
\mathrm{PR}=\mathrm{PI}=(\mathrm{IR}) \sin 45^{\circ}=.707(\mathrm{IR}), \\
\mathrm{OP}=\mathrm{OI}-\mathrm{PI}, \\
\tan \theta=\frac{\mathrm{PR}}{\mathrm{OP}}=\frac{.707(\mathrm{IR})}{1-.707(\mathrm{IR})},
\end{gathered}
$$

when OI is set to a value of 1.0 and OT or IR represent the displacement ratio. Thus the limiting values of $\theta$ are $2.1^{\circ}$ (for $5 \%$ DR) and $21.5^{\circ}$ (for $40 \%$ DR). The calculation of the angle $\theta$ for Condition 3 is the same. At threshold values of 19.7\% DR for Condition 3 and $24.3 \%$ DR for Condition 6, the angles $\theta$ amounted to $9.2^{\circ}$ and $11.7^{\circ}$, respectively.

Figure 2B shows how the oblique target motion condition differs from the orthogonal target motion condition. In the case of oblique target motion, the congruent component of the target image motion is not equal to the extent of the eye movement. Rather, it differs from the extent of the eye movement by the distance PI. Since PI equals IR times .707 , and IR, being equal to OT, represents the displacement ratio of the target motion, the congruent component of the target image motion is equal to .707 times the displacement ratio of the oblique target motion. ${ }^{6}$ At the threshold of $24.3 \%$ DR for Condition 6, the congruent component of the target image motion therefore amounted to $17.2 \%$ DR. In the analogous condition with vertical eye movements, Condition 3, the congruent component amounted to $13.9 \%$ DR. Analogous computations can be performed for Conditions 7 and 8 , where the eye movements were oblique and the target motions were either vertical or horizontal and therefore also formed an angle of $45^{\circ}$ with the plane of the eye movement. The congruent components of the target image motions can be found in Table 2, which lists, for each condition, the displacement ratio of the congruent component of the target image motion and the angle $\theta$, both at target motion threshold. There were, all told, four conditions $(6,3,7$, and 8$)$ in which the target image motion formed an angle of $45^{\circ}$ with the plane of the eye movement, and where congruent components of the target image motion occurred. The displacement ratios for these congruent components at threshold did not differ much. They ranged from $13.9 \%$ to $17.8 \%$.

The discrimination of the three general target motion conditions, congruent target motion and target motion orthogonal and oblique to the plane of the eye movement, then, results from the combination of two sensory conditions. One is the presence or absence of a discrepancy between the plane of the target image path and the plane of the eye movement, in other words, whether or not the angle $\theta$ is greater than a certain threshold value. The second factor is the difference between the extent of a congruent target image displacement (or of the congruent component of the target image displacement) and the extent of the eye movement, a difference that is discovered by the compensating process. The first factor, the presence of an angle $\theta$, is the indicator for orthogonal and oblique target motion. The second factor, the result of the compensating process, is the indicator for congruent and for oblique target motion. Hence, the three kinds of target motion are recognized by the following means: congruent target motion by the compensating process, orthogonal target motion by $\theta$ detection, and oblique target motion by both.

One may ask which contributes more to the target motion thresholds, the comparison of the extent of

Table 2

Amount of Target Image Motion in the Plane of the Eye Movement and Angle $\theta$, at Target Motion Threshold

\begin{tabular}{cccccc}
\hline $\begin{array}{c}\text { Condi- } \\
\text { tion }\end{array}$ & $\begin{array}{c}\text { Eye } \\
\text { Move- } \\
\text { ment }\end{array}$ & $\begin{array}{c}\text { Target } \\
\text { Motion }\end{array}$ & $\begin{array}{c}\text { Threshold } \\
\text { (\%DR) }\end{array}$ & $\begin{array}{c}\text { Congruent } \\
\text { Cotion } \\
\text { (\%DR) }\end{array}$ & $\begin{array}{c}\text { Angle } \theta \\
\text { (deg) }\end{array}$ \\
\hline 5 & H & H & 8.2 & 8.2 & 0.0 \\
1 & V & V & 9.3 & 9.3 & 0.0 \\
4 & H & V & 25.3 & & 14.2 \\
2 & V & H & 23.3 & & 13.1 \\
6 & H & O & 24.3 & 17.2 & 11.7 \\
3 & V & O & 19.7 & 13.9 & 9.2 \\
7 & O & V & 21.1 & 14.9 & 9.9 \\
8 & O & H & 25.2 & 17.8 & 12.2 \\
\hline
\end{tabular}


the congruent component of the target image displacement with the extent of the eye movement or the discrepancy between the plane of the target image path and the plane of the eye movement, the presence or absence of an angle $\theta$. This question can be answered by comparing the threshold values for Conditions 5 and 1, where the target motion was congruent with eye movements, with the congruent motion components computed for the thresholds for Conditions 6, 3, 7, and 8, where the target image motion was oblique to the plane of the eye movement. As stated, the displacement ratios for these congruent components ranged from $13.9 \%$ to $17.8 \%$. They all were larger than the congruent motion at threshold of $8.2 \%$ and $9.3 \%$ DR in Conditions 5 and 1 , where the threshold represents only the detection of the congruent motion. In fact, the displacement ratios of the congruent components at threshold were so much larger for these four conditions that it seems unlikely that they contributed substantially to the threshold values. It seems that the threshold for these four conditions is primarily a matter of $\theta$ detection.

There are, then, six conditions altogether where the thresholds reflect $\theta$ detection, the four conditions just discussed and, of course, the two orthogonal conditions, 2 and 4 , where there were no congruent displacement components at all. By looking at the angle $\theta$ at threshold listed in Table 2, we can obtain an idea of the accuracy with which the presence of an angle $\theta$ is detected. The value of the angle $\theta$ at threshold ranged from $9.2^{\circ}$ to $14.2^{\circ}$, with an average of $11.7^{\circ}$.

We have no explanation for the poor performance of our subjects in Condition 9, where eye movements were oblique and the target displacement congruent with the eye movement. An obvious explanation, namely, that oblique eye movements are of a different nature from those in the cardinal directions, seems ruled out by our control experiment.

The result of our analysis showed that objective target motion that is oblique or orthogonal to the eye movement is given by means of the presence of an angle $\theta$ with or without a diminished, that is, $a b$ normal, congruent target image motion component. This raises an interesting question. What enables the nervous system to make use of these properties of the target image motion path? Is an innate arrangement responsible or do we deal with a learned capacity? To propose the latter would require that conditions occur under ordinary circumstances that could yield such learning. That is, indeed, the case when, in a bright visual field, two objects move in different directions, object $\mathrm{A}$, for instance, horizontally and object $B$ with a slant. When the eyes pursue object $A$, object $B$ is given to the eye in a manner resembling the target motion of our Condition 6 . The retinal motion path of $B$ will be the resultant of the displacement of its image due to the horizontal eye movement and the displacement due to its objective motion. The resultant image motion path would be characterized by the simultaneous presence of an angle $\theta$ and of an abnormal congruent motion component. In this case, however, the objective motion path of $B$ is also independently given, by its displacement relative to the stationary objects that are visible in the bright visual field. This object-relative displacement of B leads to correct perception of its oblique motion path. It is on such occasions that a connection can become established between the simultaneous presence of an angle $\theta$ and of an abnormal congruent motion component, on the one hand, and a perceived oblique motion, on the other.

\section{REFERENCES}

KaUfman, L. Sight and mind. New York: Oxford University Press, 1974.

Kling, J. W., \& Riggs, L. A. Experimental psychology. New York: Holt, Rinehart, \& Winston, 1971.

MACK, A. An investigation of the relationship between eye and retinal image movement in the perception of movement. Perception \& Psychophysics, 1970, 8, 291-297.

WALLACH, H., \& Kravitz, J. H. The measurement of the constancy of visual direction and of its adaptation. Psychonomic Science, 1965, 2, 217-218.

Wallach, H., \& Kravitz, J. H. Adaptation in the constancy of visual direction tested by measuring the constancy of auditory direction. Perception \& Psychophysics, 1968, 4, 299-303.

Wallach, H., \& LEWIs, C. The effect of abnormal displacement of the retinal image during eye movements. Perception \& Psychophysics, 1966, 1, 25-29.

\section{NOTES}

1. Congruent,as here used, means coinciding with regard to direction only.

2. We had previously attempted, without success, to demonstrate adaptation to abnormal image displacement. We concluded that it is, at best, a slow process and would not interfere with our experiment.

3. Technical information on the SGH/V-2 may be had by writing to the manufacturer, Biometrics Inc., $\mathbf{4 0}$ Ames Street, Cambridge, Massachusetts 02142. Additional information on the application of this type of eye-movement monitor for experiments such as this will be supplied by the authors upon request.

4. In pilot research, subjects were required actually to make this comparison; when we discovered that instructions alone were sufficient to produce natural, replicable saccadic movements, we eliminated the comparison task.

5. In the experiment of Wallach and Lewis, image shifts during eye movements were always congruent.

6. This is correct only for the oblique target motions used in our experiment; their congruent component was always in the direction with the eye movement.

(Received for publication April 24, 1978; revision accepted July 27,1978 .) 